\title{
La evolución de un mismo cantar heroico desde su transmisión oral a su transmisión escrita: el caso del Cantar de Hildebrando alemán
}

\author{
The Evolution of a Heroic Lay from an Oral \\ to a Written Transmission Form: The Case \\ of the German Lay of Hildebrand
}

Miguel Ayerbe Linares

Universidad del País Vasco

Paseo de la Universidad, 5. Vitoria, 01006

miguel.ayerbe@ehu.eus

Orcid ID 0000-0002-0999-1969

Resumen: El cantar de Hildebrando nos ha sido transmitido en dos momentos temporales: primero, durante el periodo del alto antiguo alemán (750-1150), y, más tarde, durante el periodo del alto alemán paleomoderno (1350-1750). La diferencia temporal entre ambos no solo muestra cambios en la lengua alemana, desde una perspectiva puramente lingüística, sino también diferencias significantes en el modo en el que esta misma historia es narrada y transmitida. Cuando tiene lugar la primera constancia del cantar heroico de Hildebrando (Älteres Hildebrandslied-cantar antiguo) durante la primera mitad del siglo Ix, el texto muestra muchos rasgos de la poesía germánica oral tradicional, como, por ejemplo, el denominado lenguaje oralformulaico, aún muy extendido en aquel tiempo. Más tarde, durante el alto alemán paleomoderno,
RECIBIDO: 9 DE MARZO DE 2020 ACEPTADO: 26 DE AGOSTO DE 2020 la misma historia de Hildebrando y su hijo (Jüngeres Hildebrandslied-cantar moderno) se presenta en un formato literario bien diferente, que apenas muestra rasgos de la tradición oral previa. Basándonos en estos hechos es posible trazar la evolución de un mismo relato desde un formato de transmisión oral a otro de carácter escrito. Sirviéndonos de un análisis comparado, en el presente trabajo se examina cómo la transmisión de esta historia evoluciona desde un formato oral a otro escrito, es decir, cómo los rasgos propios del lenguaje oral-formulaico del cantar antiguo desaparecen en el moderno sin apenas dejar huella.

Palabras clave: Poesía heroica del alto antiguo alemán. Literatura del alto alemán paleomoderno. Oralidad. Literalidad. Lenguaje formulaico. 
Abstract: The lay of Hildebrand was handed down to us at two different points in time: the first one during the Old High German period (750-1150), and the second one during the Early New High German period (1350-1750). This difference in time is not only evident in many changes in the German language itself simply from a linguistic point of view, but also reveals significant differences in the way this story is narrated and transmitted. When the heroic lay of Hildebrand (Älteres Hildebrandslied-old lay) first appeared during the first half of the $9^{\text {th }}$ century, the text contained many features of the traditional old Germanic oral poetry, like the so called oral-formulaic language, still well-known at that time. Later on, during the Early New High German period, the same story of Hildebrand and his son (Uüngeres Hildebrandslied-modern lay) was presented in quite a different literary form, which hardly bears any traces of the previous oral tradition. On this basis it is possible to trace the evolution of the same story from oral transmission to a written (literate) form. Using a comparative analysis, this chapter examines how the transmission of this story changes from an oral to a written form, as well as the way oral-formulaic features disappear leaving almost no trace.

Keywords: Old High German Heroic Poetry. Early New High German Literature. Orality. Literacy. Formulaic Language.

\section{INTRODUCCIÓN}

L

a literatura en lengua alemana ha recogido en épocas diferentes la historia del reencuentro entre dos héroes, Hildebrando y su hijo Hadubrando. ${ }^{1}$ El primer texto, comúnmente conocido como (Älteres) Hildebrandslied (Cantar [antiguo] de Hildebrando), fue compuesto oralmente en algún momento entre los siglos V y VIII (Kartschoke 130), y puesto por escrito en el siglo IX (Bergmann 171; Classen 361, Düwel 1240); ${ }^{2}$ el segundo, denominado füngeres Hildebrandslied (Cantar moderno de Hildebrando), está recogido en manuscritos de los siglos XV y XVI. ${ }^{3}$ Como decía más arriba, ambos textos describen el reencuentro de un padre que, después de muchos años, vuelve a verse frente a frente con su hijo, a quien reconoce en algún momento del reen-

1. Hay que puntualizar que este motivo no aparece recogido en la literatura alemana medieval, exclusivamente, sino que también aparece en la literatura en antiguo nórdico, concretamente, en la Ásmunsar saga kappabana, así como también en la Pið̌reks saga, ambas del siglo XIII.

2. El texto se conserva en el manuscrito $2^{\circ}$ Ms. theol. 54, 1r, 76v de la Universitätsbibliothek de Kassel (Alemania). URL: https://orka.bibliothek.uni-kassel.de/viewer/image/1296741113093/1/ (consulta: 25.09.2019). De acuerdo con A. Classen (360), consta que solo ha sido puesto por escrito una única vez.

3. Se conservan diversas versiones del cantar moderno: por un lado, versiones fragmentarias, como el manuscrito del año 1459 en la Staatsbibliothek de Berlín, con la signatura Ms. germ. qu 1107, $52 \mathrm{v}-53 \mathrm{r}$, conocido como versión v; otro manuscrito de la Österreichische Nationalbibliothek con la signatura lat. cod. 4889, denominado X; también se conserva el fragmento del Dresdner Heldenbuch, conocido como fragmento D, del que es autor Kaspar von der Rhön. El manuscrito, datado entre 1472 y 1475 , se encuentra en la Staats- und Universitätsbibliothek Dresden, con la signatura Mscr. M 201, 345r-349r. Hay versión impresa por Valentin Newher, en Núremberg, del año 1570, conservada en la Staatsbibliothek de Berlín, con la signatura Yf8215, también incompleta. 
cuentro. Clave para ello es, en el caso del cantar antiguo, el diálogo que tiene lugar entre ambos al principio, al situarse cara a cara como caudillos de sendas huestes, en el que el padre interroga al hijo acerca de su linaje. No obstante, el hijo no reconoce a su padre, debido probablemente a los largos años de separación, y se basa en relatos orales de terceros para afirmar que su padre está muerto. ${ }^{4}$ A pesar de que el padre intenta ganarse la confianza de su hijo, si bien en ningún momento da a conocer su propio nombre, este no hace más que desconfiar y adoptar un tono cada vez más hostil, lo cual va conduciendo la situación inevitablemente hacia un duelo. Ante lo irreversible de la situación, el padre se ve enfrentado al destino de tener que decidir entre matar a su propio hijo o caer derrotado y muerto, víctima de este, ya que la renuncia al combate no es una alternativa honrosa para un héroe germánico.

Hasta aquí coinciden ambos textos. Sin embargo, aparte de la diferencia temporal entre ambos, de siete siglos como mínimo, el cantar antiguo se interrumpe en la descripción del combate entre padre e hijo, de manera que no es posible conocer cuál es el desenlace de este. En cuanto al cantar moderno, el interrogatorio del hijo por parte del padre tiene lugar en un momento del combate en el que este parece haber dominado a su hijo. Es entonces cuando ambos se dan a conocer mutuamente, mencionando sus nombres y reconciliándose. Tras esto, el hijo conduce a su padre hasta donde se encuentra su madre.

$\mathrm{Al}$ margen de las lógicas diferencias lingüísticas entre uno y otro texto, así como en el desarrollo de la narración en sí de los hechos, es posible observar también una transformación que ha sufrido lo que podríamos denominar el motivo narrativo (el reencuentro entre padre e hijo y el conflicto que se genera), desde el cantar antiguo al moderno. Dicha transformación se observa en los cambios que afectan al modo de narrar una misma historia que surgió de manera oral para ser transmitida en el tiempo. Efectivamente, al leer detenidamente ambos textos y compararlos entre sí, llama la atención que el cantar antiguo muestra claramente características propias de la literatura épica oral germánica, mientras que en el cantar moderno dichas características se hallan prácticamente ausentes. En otras palabras, en el caso del texto moderno, no se trata de una copia adaptada y/o modernizada del texto antiguo, al objeto de una mejor conservación de la historia original, realizada unos siglos después,

4. dat sagetun mi seolidante / westar ubar wentilseo, dat inan wic furnam: / tot ist Hiltibrant, Heribrantes suno (vv. 42-44) "Esto me dijeron unos marineros / que vinieron hacia el Oeste por el gran mar: / muerto está Hildebrando, hijo de Heribrando". 
sino de una transformación del carácter mismo de dicha historia y de su esencia oral, que la hace parecer un texto compuesto por escrito por primera vez, al mismo tiempo que un texto que, aparentemente, apenas tiene que ver con el cantar antiguo.

Hay que llamar la atención sobre la extensa bibliografía académica en torno a las versiones alemanas del Cantar de Hildebrando, tanto la antigua (Russom, Renoir 1988, 1977, Ebel, Schröder, Rosenfeld, entre otros) como la moderna (Heinzle, Classen, Wolff, entre otros). No faltan tampoco estudios de carácter comparativo entre ambas versiones (Hottenstein, Wolff y Heusler), así como con versiones del cantar en otras literaturas germánicas antiguas, como la nórdica (Wolff, de Boor y Kienast).

La literatura alemana medieval ha sido con frecuencia objeto de estudio desde la perspectiva de la oralidad (Oral Theory), marco del presente trabajo. Muestra de ello son los estudios y reflexiones de Haferland, Müller, Haymes, Miklautsch, Bäuml, Curschmann (1979), Wishard, Schwarz, los cuales tienen en común centrarse en la literatura en alto alemán medio, es decir, en la producción literaria a partir del siglo XI. Algunos de ellos delimitan algo más su objeto de estudio, como es el caso de Wishard (1972), que se concentra en la literatura juglaresca o Spielmannsepik, mientras que Haferland y Curschmann (1979) se centran en el Cantar de los Nibelungos.

En cuanto a la literatura alemana del periodo anterior (alto alemán antiguo 750-1050), marco temporal en el que se sitúa el cantar antiguo de Hildebrando, consta igualmente la existencia de estudios teóricos desde la perspectiva de la oralidad, como en el caso del alto alemán medio (1050-1350). Ejemplos de ello son los trabajos de Russom, Renoir (1988, 1977), Ebel y Schneider. Con todo, estos estudios no constituyen un fenómeno aislado en alto alemán antiguo, sino que también existen en el ámbito del inglés antiguo (O’Brien O'Keeffe, Schaefer, Parks y Green), y en la literatura en antiguo nórdico (Harris 2010 y Kellogg).

Ahora bien, a pesar de la bibliografía existente en torno a un análisis comparativo entre el cantar antiguo y el moderno, así como también acerca de características de oralidad en el cantar antiguo, se echa en falta estudios acerca de cómo evoluciona el modo de narrar la historia y su forma, desde el cantar antiguo al moderno. Más concretamente, hasta donde he podido buscar, faltan estudios que analicen y describan en qué aspectos concretos se va reconociendo que el modo de narrar la historia del reencuentro entre padre e hijo ha ido abandonado su carácter oral hasta llegar a transformarse en un texto de ca- 
rácter escrito, tal como indicaba más arriba. Faltan respuestas a preguntas como, por ejemplo, qué elementos formales concretos y característicos del cantar de gesta germánico, qué rasgos de oralidad, presentes en el cantar antiguo, han desaparecido en el modo de narrar la historia en el cantar moderno; qué elementos discursivos presentes en el cantar antiguo han sufrido una importante modificación o, incluso, han llegado a desaparecer por completo en el cantar moderno.

\section{Objeto DEL PRESENTE TRABajo}

A la vista de lo dicho en el apartado anterior, en este estudio me centraré en el análisis comparado de ambos textos del cantar (antiguo y moderno) en lengua alemana, al objeto de poner de manifiesto en la medida de lo posible las diferencias en la forma de narrar un mismo contenido temático, desde un carácter eminentemente oral hasta otro de carácter escrito. Para ello, parto de la hipótesis de que en el cantar antiguo está presente una serie de características formales y discursivas propias de la literatura heroica de transmisión oral, que van desapareciendo con el paso del tiempo hasta llegar al punto de brillar por su ausencia en el cantar moderno. A consecuencia de ello, el cantar moderno mostraría entonces una serie de características formales y discursivas propias de un lenguaje que, aun siendo todavía épico, corresponde a un mundo distinto, esto es, al cortesano-caballeresco con sus características propias.

Ahora bien, hablar de características formales propias de la literatura heroica de transmisión oral presupone, en primer lugar, conocer qué se entiende exactamente por tales. Y es aquí donde hay que decir que, afortunadamente, existe una extensísima producción académica en torno al concepto de literatura oral y sus características. Milman Parry (1928a; 1928b; 1930) es comúnmente considerado el precursor de la teoría en torno a la poesía oral (Oral Poetry) en la primera mitad del siglo Xx (Foley/Ramey 2016, 75; Dunphy 105; Kartschoke 39; Windelberg/Miller 29; Curschmann 1977, 64), aplicándola al análisis de epítetos en la poesía de Homero, donde ya habla del concepto de "fórmula (oral)" (1928a/b). Es conocida su definición clásica de "fórmula" como "une expression qui est régulièrement employée, dans les mêmes conditions métriques, pour exprimer une certain idée essentielle" (1928a, 16), la cual modificó él mismo un par de años después en términos tales como "a group of words which is regularly employed under the same metrical conditions to express a given essential idea" $(1930,80)$. 
A las definiciones de Parry siguieron otros estudios, cuyo objeto consistió en seguir desarrollando el concepto ${ }^{5}$ y en aplicarlo a literaturas de la Edad Media producidas en otras lenguas, tanto germánicas (Ford, en este volumen; Murray, en este volumen; Müller; Lord 1995; 2000; Andersson; Mitchell; Parks; Bäuml; Riedinger; Harris 1983; Wishard; Fry 1981; 1968; Lönnroth 1981; 1971; Curschmann 1967; Jones; Schwarz; Whallon; Greenfield; Magoun), como románicas (Ashby-Beach; Webber 1980; Duggan; Hackett; Waltman; Adams; Aguirre; Curschmann 1967; Webber 1951). También la literatura en lenguas eslavas ha sido objeto de estudio en este terreno (Lord 1995; Foley 2000; 1983).

A los estudios anteriormente mencionados hay que añadir aquellos otros que se han ocupado de revisar el concepto mismo de fórmula, como Windelberg y Miller, Kiparski, Nagler y Fry (1967), entre otros. A modo de ejemplo, cabe citar aquí la definición propuesta por este último para la poesía en inglés antiguo:

a group of half-lines, usually loosely related metrically and semantically, which are related in form by the identical relative replacement of two elements, one a variable word or element of a compound usually supplying the alliteration, and the other a constant word or element of a compound, with approximately the same distribution of non-stressed elements. $(1967,203)$

Finalmente, cabe citar también la definición de Windelberg/Miller, según la cual "the formula is a collocation of words which primarily occurs as sister constituents of a sentence. The elements of the collocation are bound together by co-occurrence expectancies that exhibit varying probabilities" (47).

Con todo, hay que añadir que, si bien los comienzos de la teoría de la oralidad como tal se remontan a Milman Parry, conceptos y elementos de la poesía oral como fórmula, epiteto o repeticiones, entre otros, ya habían sido objeto de análisis con anterioridad. Baste mencionar, a modo de ejemplo, el extenso trabajo de Meyer aplicado a las literaturas germánicas antiguas, el cual, a su vez, hace alusión a otros trabajos previos. Es cierto que Meyer no pretende elaborar un compendio acerca de lo que más tarde se llamaría la teoría de la poesía

5. Por razones de espacio, no puedo extenderme aquí en el desarrollo histórico del concepto de "fórmula". No obstante, para una visión panorámica de carácter histórico, puede consultarse Foley, 1981 y 1985, así como Windelberg/Miller. 
oral y las fórmulas orales a partir de Parry, además de que se ocupa de elementos que hoy en día no estarían dentro de dicha teoría, pero se le puede considerar de alguna manera un iniciador. Al mismo tiempo, ya adelanta, si bien en un tono aún muy genérico, lo que ocurre en la transición de la composición oral a la escrita:

Wie die poetische Sprache der allgemeinen, so wächst die Schriftsprache der gesprochenen nach. Erst fallen die Worte aus als nicht schriftgemäss; dann Wendungen, Satzformen u. s. w. Schliesslich ist sogar die Art der Eintheilung des Ganzen bestimmt: Capitel, Abschnitte, Absätze folgen einer allgemeinen Convenienz. Bei zunehmender Erstarkung kann so alle Form mechanisirt werden. (Meyer 12)

Esto último se cumple en el caso del cantar antiguo de Hildebrando, y para comprobarlo basta con acudir a una imagen del manuscrito en el que se conserva el cantar, en lugar de consultar ediciones más modernas del texto. Allí se constata, por ejemplo, que el cantar no está dispuesto en versos listados individualmente, como suele ser el caso de las ediciones de poesía. En el manuscrito, los versos están concatenados en líneas y no se hallan visiblemente fragmentados por la cesura. Si se acaba el espacio de una línea, el escriba continúa lo que nosotros catalogamos como verso en la línea siguiente. Ver a modo de comparación la tabla 1, con la reproducción del comienzo del cantar antiguo, en el manuscrito (columna izquierda) y en una edición moderna (columna derecha):

Manuscrito Kassel $2^{\circ}$ Ms. Theol. fol. 1r Edición según Braune/Ebbinghaus (1994)

Ik gihorta đat seggen đat sih urhettun ænon muo Ik gihorta đat seggen, tin hiltibrant enti hađubrant untar heriun tuem. sunufatarungo iro saro rihtun garutun se iro guđhamun gurtun sih iro suert ana helidos ubar ringa. (líneas 1-5)
dat sih urhettun ænon muotin,
Hiltibrant enti Hađubrant untar heriun tuem. sunufatarungo iro saro rihtun. garutun se iro guđhamun, gurtun sih iro suert ana, helidos, ubar bringa. (versos 1-6)

Tabla 1. Comparativa del comienzo del cantar antiguo en el manuscrito y en una edición moderna.

La indudable ventaja de escoger para el presente trabajo la historia del reencuentro de Hildebrando y su hijo Hadubrando radica, como decía más arriba, en la circunstancia de contar con una misma historia que nos ha sido legada en dos situaciones temporales distintas, lo cual nos permite observar las dife- 
rencias formales a la hora de narrar dicha historia y constatar, en su caso, los cambios que se han producido en el modo de transmitir la historia.

Procedo a continuación al análisis de los dos cantares. Para el del cantar antiguo me he basado, por un lado, en el manuscrito conservado en la biblioteca de Kassel, al que ya me he referido anteriormente, por otro lado, en el texto editado por Braune/Ebbinghaus en 1994, teniendo en cuenta la alteración de forma que estos realizan, al presentar el texto en forma versificada, y no en líneas, como ocurre en el manuscrito. ${ }^{6}$ En cuanto al cantar nuevo, he utilizado como base la edición de von der Hagen/Primisser, de 1825, basada en el Dresdner Heldenbuch (h. 1472).7 Se trata de la versión D, la más extensa en 29 estrofas de 8 versos cada una.

El análisis de ambos cantares lo efectuaré por separado. En primer lugar, me ocuparé del cantar antiguo, tratando de poner de manifiesto los rasgos de carácter oral que se hallan presentes. En la medida de lo posible, procuraré situar dichos rasgos en el contexto germánico antiguo, acudiendo a citas de textos literarios compuestos en otras lenguas germánicas antiguas. Esto último será útil en algunas ocasiones, al objeto de reforzar el carácter de fórmula de algunas expresiones. En un segundo lugar, abordaré al cantar moderno, intentando llamar la atención sobre la ausencia de los rasgos de oralidad constatados en el cantar antiguo, así como los rasgos propios del cantar moderno. En cuanto a la fuerte presencia del lenguaje cortesano en el cantar moderno, por razones de espacio me vi obligado a no abordarlo aquí, ya que, a pesar de su eventual relevancia para mostrar el distanciamiento del lenguaje oral-formulaico del cantar antiguo, quedaría fuera de lo que constituye el objeto del presente análisis. Finalmente, en las conclusiones, resumiré las principales diferencias observadas entre las dos versiones de la historia entre Hildebrando y Hadubrando a la luz del análisis efectuado.

\section{El cantar antiguo}

\subsection{Fórmulas sintácticas}

a) Uso del pronombre personal de tercera persona singular masculino (her) + verbo. En pocos versos se repite una misma estructura oracional, incoada por

6. Para traducciones al español se puede consultar la de B. Raposo y la de F. Díaz.

7. El Heldenbuch se conserva en la Sächsische Landesbibliothek - Staats- und Universitätsbibliothek Dresden, con la signatura Mscr. Dresd. M. 201. El texto del cantar moderno de Hildebrando está localizado entre 345r y 349r. 
el pronombre personal her ('él'), lo cual otorga cierto ritmo a la narración. Para una mayor visibilidad, retiro la cursiva en los versos en cuestión:

her furlaet in lante luttila sitten prut in bure, barn unwabsan, arbeo laosa: ber raet ostar bina. des sid Detribhe darba gistuontun fateres mines: dat uuas so friuntlaos man. her uuas Otachre ummet tirri, degano decbisto miti Deotribhe. her uuas eo folces at ente. (20-27)
"Él dejó abandonados en su tierra a la esposa en casa y a un niño muy pequeño, sin herencia, y marchó hacia el Este. Teodorico tuvo gran necesidad de mi padre; era un hombre sin amigos.

Él tenía un gran odio a Odoacro. El más fiel de los guerreros al lado de Teodorico, él estaba siempre al frente de la tropa".

Como se puede observar, en los dos últimos casos también la forma verbal (uuas) coincide.

La repetición puede tener lugar también dentro del mismo verso, quedando unidos ambos hemistiquios por yuxtaposición:

forn her ostar giweit, flob her Otachres nid. (18)
"Hace tiempo marchó él al Este, huyó él del odio de Odoacro".

b) Concatenación de proposiciones subordinadas sustantivas de objeto directo, introducidas por la misma conjunción (dat) y con el mismo sujeto:

wela gisibu ib in dinem brustim, dat du babes beme herron goten, dat du nob bi desemo riche reccheo ni wurti. (46-48) "bien puedo reconocer por tu armadura que tienes un buen señor en tu patria, que bajo su reinado nunca padeciste exilio".

c) Repetición de la estructura compuesta por un sintagma nominal en función de objeto directo + verbo en infinitivo, con el mismo significado semántico:

nu scal mih suasat chind

suertu bauwan,

breton mit simu billiu. (53-54)

in sus heremo man brusti givinnan, rauba birabanen. (56-57) "y ahora mi querido hijo habrá de matarme con la espada, abatirme con su hoja afilada".

"de este hombre tan viejo conseguir la armadura, quitarle el botín".

En el primer ejemplo, la estructura sintáctica que se repite soporta el contenido semántico "matar con la espada", el cual es expresado con unidades léxicas diferentes. En el segundo, el contenido semántico es "obtener la armadura (del otro)". Esto hace que estas mismas fórmulas sintácticas puedan ser consideradas al mismo tiempo como fórmulas semánticas, ya que se repite el mis- 
mo contenido semántico, aunque sea con variación léxica. Como se puede comprobar, el contenido expresado en ambos hemistiquios es el mismo o muy parecido.

d) repetición de epítetos con la estructura: antropónimo 1 en nominativo + verbo dicendi + antropónimo 2 en genitivo + suno "hijo". Estos epítetos eran muy comunes en la literatura heroica germánica, donde la carta de presentación de un héroe determinado consistía en rememorar a sus antepasados -especialmente al padre-, de gloriosa memoria: ${ }^{8}$

Hiltibrant gimabalta Heribrantes sunu. (7, 45) "Habló Hildebrando, hijo de Heribrando".

Hadubrant gimabalta, Hiltibrantes sumu. $(14,36)$ "Habló Hadubrando, hijo de Hildebrando".

A raíz de estos ejemplos se puede constatar que el contenido referencial de los antropónimos 1 y 2 puede variar, lo cual quiere decir que nos encontramos ante dos expresiones formulaicas distintas. Sin embargo, algo que es importante es que cada expresión de este tipo sea recurrente. A este respecto, se plantea la cuestión acerca del número mínimo de repeticiones para que se pueda considerar formulaica una expresión concreta. Miletich (112), interpretando a Bäuml, habla de, al menos, dos repeticiones, criterio que se cumple en el cantar antiguo que estamos analizando aquí.

\subsection{Fórmulas semánticas}

En la literatura germánica de la Alta Edad Media es frecuente encontrarnos con repeticiones de contenidos semánticos con variación léxica. Esto es, al final de un verso y al comienzo del siguiente se repite dos veces el mismo contenido semántico, ${ }^{9}$ variando los significantes:

Hiltibrant gimabalta Heribrantes sunu:

ber uuas heroro man,

ferabes frotoro. (7-8)
"Habló Hildebrando, hijo de Heribrando: era el de más edad, el de más experiencia en la vida".

8. Un ejemplo de la literatura en inglés antiguo, concretamente, del Beowulf: Unferð mapelode, Ecgläfes bearn (499), "Habló Unferơ, hijo de Ecglāf".

9. En el Heliand, en sajón antiguo, la repetición semántica puede llegar incluso a tres veces: thar ina Iohannes antfand, / that friðubarn godes, frôhan sînan, / hêlagana hebencuning... (1127-1129) "Allî lo encontró Juan, / al hijo de la paz de Dios, a su señor, / al santo rey del cielo...”. Y lo mismo se puede afirmar en inglés antiguo: Wē purb holdne hige bläford pīnne, / sunu Healfdenes sēcean cwōmon / lēodgebyrgean (Beowulf 267-69). "Nosotros con buenas intenciones a tu señor, / al hijo de Healfden, al protector del pueblo, / venimos a ver". 
want her do ar arme wuntane bauga, Entonces se desprendió de los brazaletes trenzados cheisuringu gitan, so imo se der chuning gap, hechos con monedas que le había dado el rey, Huneo trubtin. (33-35) el señor de los hunos".

En el primer caso, el contenido semántico que se repite es "hombre de mayor edad y experiencia en la vida", y en el segundo "rey, señor". Con esta insistencia sería más fácil que la audiencia retuviera en su memoria determinados personajes y sus cualidades para estar en condiciones de recordarlos cuando volvieran a aparecer en otros pasajes del mismo relato, ya que no podían acudir a otra fuente, como un texto escrito, ya fuera por su inexistencia como por su incapacidad para leer.

\subsection{Elementos discursivos}

En primer lugar, dado que nos estamos ocupando de textos de composición oral, sería oportuno mencionar aquí la fórmula "Yo oí contar", con la que suele comenzar un relato heroico. En el cantar antiguo, esta fórmula aparece como sigue:

$$
\text { Ik giborta đat seggen. (1) "Yo lo oí contar". }
$$

Este tipo de fórmula ha sido objeto de estudio por parte de autores como Parks y Rumble en la poesía épica en inglés antiguo para designar la acción de volver a contar un relato oral previo. Ahora bien, Parks (53-54) llama acertadamente la atención sobre el hecho de que no siempre se habla de una fórmula oral en sentido propio cada vez que se emplea esta expresión en un texto épico. En consecuencia, para evitar confusiones propone la aplicación de tres criterios que ayuden a esclarecer la naturaleza de cada expresión "Yo oí contar": 1) limitar el análisis a los verbos hyran y gefrignan, como verbos canónicos objeto de estudio; 2) el narrador/sujeto de estas expresiones debe usar el pronombre personal $I$ o we para hacer referencia a sí mismo o a sí mismo y a su comunidad como narrador o fuente en el presente acto de narrar, y no como narrador en un momento pasado, dentro de la narración misma que se está ejecutando; 3 ) la fórmula "Yo oí contar" debe ser empleada para introducir y autentificar la historia que va a ser objeto de la narración. En otras palabras, debe emplearse como un modo de preparar a la audiencia para lo que va a escuchar.

En el caso concreto del cantar antiguo de Hildebrando, estos criterios de Parks resultan especialmente útiles, ya que en este texto se constatan hasta tres expresiones que hacen referencia a relatos previos, así como fuentes orales previas: 

Ik gihorta dat seggen. (1)
"Yo he oído contar".
dat sagetun mi usere liuti. (15)
"Esto me dijo nuestro pueblo".
dat sagetun mi seolidante. (42)
"Esto me dijeron unos marineros".

De las tres citas, solo la primera cumple los criterios de Parks, ya que es la única en la que el verbo bōren se corresponde con uno de los marcados (byran): empleo del pronombre $I k$ de primera persona singular nominativo, que en tiempo presente actual está contando la historia desde fuera de esta. Aparte de esto, en los casos restantes es un personaje que forma parte del relato (Hadubrando) el que se remite a fuentes orales previas (sagēn "decir, contar"), por lo que no podemos calificar ambas citas como fórmulas.

Cuestión bien distinta es que podamos considerar la estructura dat sagetun mi, del primer hemistiquio, una fórmula oral de tipo sintáctico, dada su repetición, y donde el único elemento variable estaría constituido por el segundo hemistiquio (pueblo, marineros, etc.). Dicho de otro modo, la expresión dat sagetun mi no sería una fórmula tipo "Yo oí contar" en el sentido de Parks, al no cumplir sus criterios, pero sí podría ser una fórmula sintáctica de la que se serviría el poeta para transmitir el relato, utilizada a modo de repetición. De hecho, en la literatura germánica antigua era frecuente remitirse a relatos orales de terceros, que exponían las hazañas y gestas de héroes, ya fueran del propio linaje o de otro. ${ }^{10}$

Hay, finalmente, una expresión en el cantar antiguo que, por no cumplir el criterio de frecuencia o regularidad (Lord 1995: 122, Windelberg/Miller 39), podría pasarse por alto o descartarse como expresión formulaica, ya que aparece una sola vez en el texto:

nu scal mib suasat chind

suertu hauwan. (53)

$$
\begin{aligned}
& \text { "y ahora mi querido hijo habrá de matarme } \\
& \text { con la espada". }
\end{aligned}
$$

Se trata aquí de la expresión suasat chind ("querido hijo"), la cual, casualmente, se encuentra también en la Ásmundarsaga kappabana (ss. XIII-XIV, en nórdico antiguo), donde el mismo protagonista, Hildebrando, poco antes de morir confiesa a su hermano Asmund haber sido quien, en contra de su propia voluntad, dio muerte al hijo de su misma sangre:

Liggr par inn suási sonr at böfoi. (8, III, 4) "Ahí junto a mi cabeza se halla el hijo querido".

10. Un ejemplo procedente del Beowulf: Nō ic wiht fram pēe / swylcra searonīða secgan hyrde (581-82), "Yo de ti, por el contrario, / no he oído contar tales combates". 
Encontramos, pues, una misma expresión en dos textos y contextos diferentes -recordemos que en el cantar antiguo alemán la expresión se utiliza durante la tensa conversación entre Hildebrando y su hijo Hadubrando-, obra presumiblemente de distintos poetas. En este sentido se expresaban Windelberg/Miller (39-40), al advertir del riesgo de tomar en sentido estricto el criterio de regularidad o repetición, ya que el carácter formulaico de una expresión determinada que solo aparece una vez en un texto oral puede verse confirmada como tal gracias a su uso en otros textos de similares características (Windelberg/Miller 40). A esta conclusión se puede llegar tras realizar un estudio cruzado de fórmulas a lo largo de diferentes textos, como el efectuado por Rychner en textos épicos franceses. Esto nos lleva a una conclusión digna de ser remarcada: el carácter formulaico de una expresión, entonces, no viene dado exclusivamente por el hecho de ser empleada repetidas veces en un mismo texto, sino también por el hecho de estar presente en más textos de la misma naturaleza.

Con todo, hay otro rasgo que no podemos pasar por alto, sobre todo, al hablar del cantar antiguo de Hildebrando como muestra de la poesía germánica heroica de transmisión oral: la aliteración. Se trata de un recurso de naturaleza fonológica, consistente en la repetición de un sonido inicial concreto a lo largo de un mismo verso:

Hiltibrant enti Hadubrant untar herium tuem. (3)

"Hildebrando y Hadubrando entre dos huestes".

Como se puede observar, en este verso se repite tres veces el comienzo de una palabra con la consonante [h]: Hiltibrant, Hadubrant, y herium.

Que la aliteración es un recurso especialmente apropiado para la literatura germánica de composición y transmisión oral lo afirma una extensa bibliografía académica, la cual llama la atención especialmente sobre dos aspectos: por un lado, que se trata de una herramienta con un peso que va más allá de meras consideraciones estéticas (Lea/Rapp/Elfenbein 715); por otro, que se trata de un recurso mnemotécnico especialmente útil, tanto para el poeta como para su audiencia, pero sobre todo para esta última (Boers/Lindstromberg/Eyckmans 291; Lea/Rapp/Elfenbein 709; Minkova 6). ${ }^{11}$ Hay que decir

11. Existen también numerosos estudios que, desde el siglo pasado, se han ocupado de llevar a cabo experimentos al objeto de analizar desde una perspectiva psicológica los efectos de la aliteración sobre la capacidad de memorizar texto o discurso en oyentes frente a otras técnicas sin aliteración. No obstante, debido a razones de espacio, y a que ya me estaría desviando del tema de estudio en el presente trabajo, no puedo extenderme ahora en ello. 
que la aliteración, como recurso literario, centra más la atención en la audiencia que en el poeta, ya que mediante unidades fonológicas busca reactivar en la memoria del oyente información ya recitada previamente, puesto que la poesía aliterativa no fue compuesta para lectores, sino para oyentes (Minkova 6). Por tanto, la aliteración puede considerarse como una señal elocuente de que un texto ha sido compuesto oralmente para ser transmitido de forma oral a un público que no lo va a tener delante por escrito.

Finalmente, quedaría un último rasgo para comentar que, desde mi punto de vista, puede resultar especialmente relevante para resaltar el carácter de oralidad del cantar. Me estoy refiriendo aquí al modo en el que este nos ha llegado. Efectivamente, el texto del cantar se ha conservado en la primera (1r) y última (76v) páginas del códice, y no dentro del cuerpo del códice mismo, lo cual resulta especialmente llamativo. De hecho, esta circunstancia explica, a mi modo de ver, que el cantar no se haya conservado íntegro, ya que el escriba, una vez que se le acabó el espacio, interrumpió la narración sin más, tal como se puede observar en la última página, en la imagen 1 :

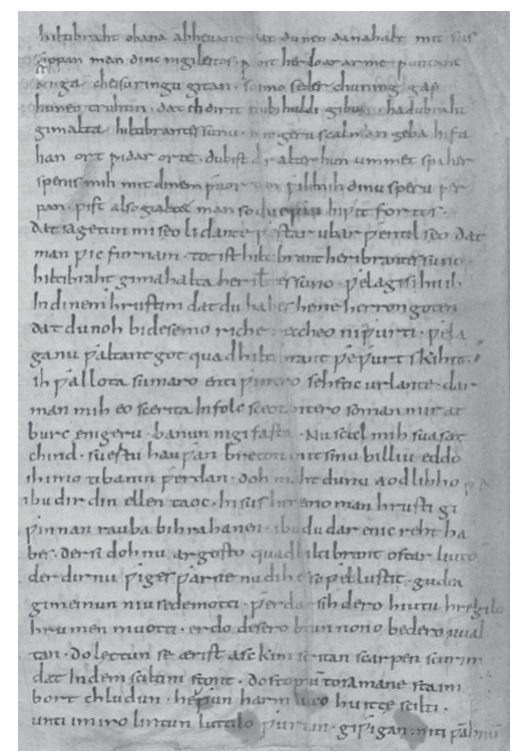

Imagen 1. Segunda parte del cantar, según el $2^{\circ}$ Ms. Theol. fol. 54, 76v de la Universitätsbibliothek Kassel, Landesbibliothek und Murhardsche Bibliothek der Stadt Kassel (Alemania). ${ }^{12}$

12. URL: https://orka.bibliothek.uni-kassel.de/viewer/image/1296741113093/156/LOG_0012/ (Consulta: 23.10.2019). 
Ahora bien, la razón por la que la puesta por escrito del cantar quedó inconclusa sigue constituyendo hoy en día un interrogante abierto. Podría deberse a la pérdida de una hoja adicional, o bien a que los escribas dejaron de escribir una vez se les acabó el espacio, debido probablemente a lo extendido que debía estar el cantar oralmente.

Ahora bien, lo que me parece realmente relevante al mirar detenidamente el manuscrito en el que nos ha llegado el texto es que podemos descubrir lo que podría denominarse una marca extralingüística de oralidad. La clave para entender en qué consiste se halla, entre otras cosas, en lo que algunos estudiosos de este cantar antiguo llaman Editionsprobleme, "problemas de edición" (Düwel/Ruge 2013, 172-73; Düwel 1241-42), al hablar de aspectos como puntuación, separación de líneas, palabras, espacios, etc. Por la forma en la que el cantar nos ha llegado, dichos autores se encuentran con grandes dificultades, por ejemplo, a la hora de determinar límites entre oraciones, espacios entre palabras, así como al considerar una secuencia de caracteres como una única palabra o como palabras separadas. Y es que realidades como los espacios, tan nítidas para nosotros en la actualidad, en aquel entonces podían tener alguna explicación más dudosa como marca de separación de palabras en un texto escrito. Por mencionar un caso concreto, Düwel/Ruge (172), citando a otros autores, llaman la atención sobre las diversas posibilidades que plantea la secuencia ummettirri (verso 25 en la edición de Braune/Ebbinghaus, línea 20 en la primera hoja del manuscrito), a la hora de editar con posterioridad el cantar: ummet tirri, ummett irri y, finalmente, ummet irri.

Hechos como este son frecuentes en textos antiguos compuestos oralmente que, posteriormente, son puestos en algún momento por escrito en un contexto en el que la escritura tiene un papel poco relevante, y en un momento histórico en el que en una sociedad las manifestaciones literarias aún no han conocido la transmisión por escrito, la cual presenta forzosamente unas características formales muy distintas de la composición y transmisión orales. A este respecto, Dunphy lo describe de manera muy ilustrativa:

In a society without writing, words are events and cannot be visualized spatially. The poet may arrange words in lines, but has no clear concept of a "word" and none at all of a "line" - language can only be linear when it is written. Without a system of writing, poetry is performance and can- 
not be pinned down to a permanent state in which it can be analyzed. Our concept of "text" as a fixed corpus of words makes no sense in an oral culture, since there is no way to check whether two recitations are identical. (104)

$\mathrm{Al}$ mismo tiempo, hay que llamar también la atención sobre el hecho de que la redacción del texto aglutine rasgos de al menos tres lenguas germánicas antiguas: antiguo alto alemán (ib 17, prut 21, tot 44), sajón antiguo (Ik 1, usere 15) y langobardo (antropónimos Hildebrand, Hadubrand y Heribrand). Si bien no es raro encontrar variaciones grafemáticas en un mismo texto de esta época, estas se deben con frecuencia a variaciones dialectales o a vacilaciones por parte del escriba, ya que este podía ser de un origen distinto al del lugar donde se encontraba en ese momento. ${ }^{13}$ En estas circunstancias, es de suponer que el escriba no dominaría bien la lengua del lugar. En cualquier caso, circunstancias como esta pueden indicar un origen que no había sido compuesto para una transmisión por escrito.

\section{El CANTAR MODERnO}

$\mathrm{Al}$ margen de conocer el desenlace del reencuentro de Hildebrando con su hijo, este texto presenta una serie de cambios que podemos clasificar en dos grupos: en primer lugar, los rasgos de la poesía heroica antigua de transmisión oral, que en este texto del cantar han desaparecido; en segundo lugar, rasgos de un tipo de composición literaria más propios de una época posterior, y que tienen que ver más bien con un contexto cortesano que con el de las antiguas sagas heroicas de transmisión oral.

\subsection{Ausencia de rasgos de oralidad}

En lo que respecta al primer grupo de cambios, lo primero que llama la atención es la ausencia de referencias a fuentes orales previas, tanto del narrador externo de la historia como desde dentro, es decir, de los propios personajes. En otras palabras, no hay marca discursiva alguna que indique una tradición oral anterior ("Yo oí contar") o, mejor dicho, la recepción de una tradición oral

13. Un buen ejemplo de esto puede verse en la Oración de Wessobrunn ("Wessobrunner Gebet o Schöpfungsgedicht"). 
previa. Al contrario, el cantar comienza con unas palabras de Hildebrando, con las que expresa su deseo de volver a su tierra después de muchos años: "Ich solt $z$ u land aus reiten" / sprach meister Hildeprant (I, 1-2). ${ }^{14}$

Tampoco encontramos fórmulas sintácticas ni semánticas (repeticiones, epítetos) que indiquen ayudas para el poeta, a la hora de memorizar el cantar, así como para ayudar a su audiencia a recordar quién era quién en el texto. Por poner un ejemplo, aquí ya no se hace alusión a la ascendencia de Hildebrando ni a la de su propio hijo ("sprach [...] Hildeprant" I, 2; "Hiltprant der sprach mit sitten" v, 1), ni tampoco a la de otros personajes ("sprach Diterich von Pern" IV, 1) como sí ocurría en el cantar antiguo ("hijo de...”).

Del mismo modo, en la forma de narrar la historia ya no constatamos fórmulas semánticas consistentes en la repetición, en versos contiguos, de un contenido semántico parecido mediante diferentes unidades léxicas, como era el caso en el cantar antiguo. Tampoco se observan las repeticiones sintácticas que veíamos en el cantar antiguo, como, por ejemplo, las oraciones que comenzaban con el pronombre personal her, lo cual hace más dinámico el hilo discursivo del texto, es decir, no se vuelve una y otra vez sobre una misma idea o matiz, puesto que ya no es necesario al estar el texto fijado por escrito.

Desde un punto de vista estrictamente formal podemos constatar también la desaparición de la rima aliterada, tan frecuente en la literatura germánica antigua. Como ya expliqué más arriba, esta consistía en la repetición de un mismo comienzo fonético. Un ejemplo de ello podemos ver en la siguiente cita del cantar antiguo, con aliteración en ch-: chind, in chunincriche: chud ist mir al irmindeot (13) ("muchacho, en este reino conozco a todo el mundo"). En el cantar moderno, en cambio, la rima es final (ababcdcd) hasta tal punto que permite reconocer una estructura estrófica uniforme de ocho versos a lo largo de todo el texto:

"Ach, vater mein, die wunden
die ich dir bab geschlagen,
die wolt ich zu allen stunden
an meinem leibe tragen".
"nun schweig bie still, traut sune mein,
der wunden wirt gut rot;

"Ach, vater mein, die wunden

die ich dir bab geschlagen,

an meinem leibe tragen".

der wunden wirt gut rot;
“Ah, padre mío, las heridas

que te he causado,

ya me gustaría a mí quedármelas

y llevarlas en mi cuerpo".

"Calla, mi hijo querido,

las heridas están bien donde están;

14. En estas referencias, las cifras romanas indican el número de estrofa, y las arábigas los versos dentro de la estrofa. 
und mus got ymer gelobett sein, das keiner bie ist dot". (XVII) ¡que Dios sea siempre alabado, ya que aquí ninguno yace muerto!".

Como se puede observar en esta estrofa citada, no encontramos repetición alguna de inicios de palabra con una misma consonante, que marque además un determinado ritmo. Aquí el destinatario no es un oyente sino un lector a quien no hay que hacerle memorizar una información determinada. Puede leer lo que tiene delante y volver sobre ello más tarde todas las veces que desee, algo que no era tan fácil para una audiencia en la Alta Edad Media.

\subsection{Rasgos propios del cantar moderno}

Ahora bien, aparte de los rasgos propios de la literatura de transmisión oral que ya no se observan en el cantar nuevo, podemos constatar en este otros rasgos más propios de la literatura de transmisión escrita. Un ejemplo de ello es la presencia de hipotaxis:

Do nun der alt Hilprande

durch den rossen gartn aus reit

ind marck des Perners lande,

kom er in gros arbeit,

wol von dem inngen, mit gewalde,

do wurd er an gerant. (VI, 1-6)
"Así pues, cuando el viejo Hildebrando

llegó cabalgando desde el Rosengarten

a tierras de la marca de Verona,

se vio de repente en una situación muy difícil,

pues salió a su encuentro, muy hostil,

aquel joven".

La hipotaxis como estrategia propia de textos compuestos para su transmisión por escrito, o como característica propia de textos escritos, ha sido tratada con frecuencia. Baste consultar para este fin estudios como el de Tannen y Chafe, entre otros. Si bien es cierto que estos autores, así como otros estudios que ellos, a su vez, mencionan, centran su análisis en el contraste entre el discurso oral y el discurso escrito en la actualidad, las observaciones y conclusiones que extraen son también aplicables a los textos que estamos comparando aquí, pues se trata de la narración de una misma historia -el reencuentro de Hildebrando con su hijo tras largos años de separación-, realizada, por un lado, en forma de transmisión oral, por otro, a través de la transmisión escrita. En este sentido, los autores coinciden en la observación de que una versión escrita utiliza estructuras sintácticas más complejas, como puede ser la hipotaxis (Tannen, Chafe y Ochs).

Por último, en el códice del Dresdner Heldenbuch se pueden ver marcas que permiten identificar una estructura organizada en estrofas: 


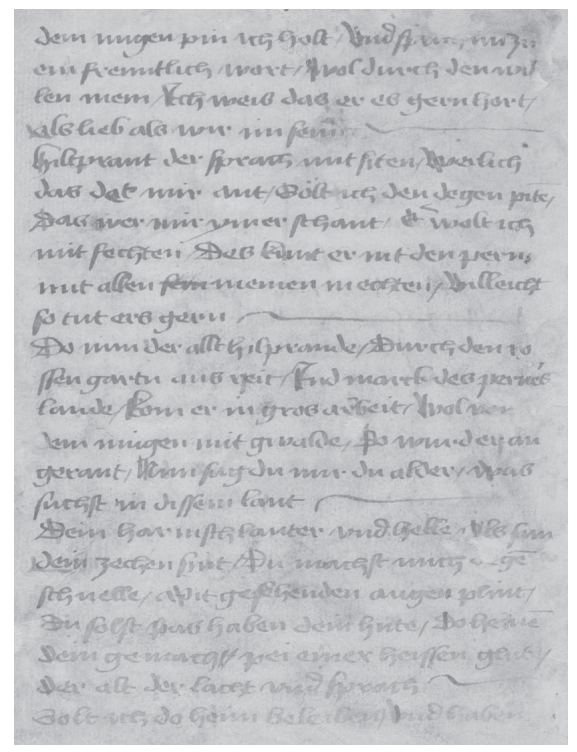

Imagen 2. Reproducción de 345v del códice Mscr. Dresd. M. 201, de la SLUB Dresden/Digitale Sammlungen.

En la imagen 2 se puede constatar la existencia de trazos curvos que cierra el último verso de una estrofa. Al mismo tiempo, puede observarse una marca más oscura en la letra inicial del primer verso de la nueva estrofa. Dicha letra aparece también escrita en mayúscula. Se trata de efectos visuales que tienen sentido para un público que va a leer el texto y no a escucharlo.

\section{CONCLUSIÓN}

Una misma historia, esto es, el reencuentro entre los héroes germánicos Hildebrando y su hijo Hadubrando se ha analizado teniendo en cuenta cómo nos ha llegado en dos puntos temporales distintos, entre los que mediaba una distancia de siete siglos. El análisis se ha centrado en las diferencias en la forma de narrar esta única historia, desde un formato propio de la literatura heroica de transmisión oral a otro de transmisión escrita, tratando de poner de manifiesto qué elementos propios de la composición y transmisión oral están presentes en el primer texto (cantar antiguo) y desaparecen más tarde en el siguiente (cantar moderno), al tratarse, como decía antes, de un texto compuesto para ser transmitido por escrito. 
El análisis realizado nos permite comprobar que los rasgos de oralidad propios de la poesía heroica germánica de transmisión oral constatados en el cantar antiguo ya no se hallan presentes en el cantar moderno. En concreto, de la aliteración, así como de la referencia a fuentes orales previas ("Yo oí contar”) ya no queda nada. Tampoco encontramos marcas de fórmulas repetitivas, tanto a nivel sintáctico como a nivel semántico, las cuales, a modo de cantinela repetitiva, se van quedando grabadas en la memoria del auditorio, tanto para reactivar informaciones previamente recitadas, como también para tenerlas en cuenta más adelante a lo largo de la recitación. Todo eso no es necesario en un texto compuesto para ser leído, es decir, visualizado, en lugar de escuchado. Al mismo tiempo, el texto moderno presenta unas características propias correspondientes a una transmisión escrita, como la hipotaxis recurrente y la organización en estrofas, que no se ve en el texto antiguo.

En otro orden de cosas, la misma transmisión del texto antiguo, que nos ha llegado en un único manuscrito, demuestra, a mi modo de ver, que para los escribas la historia no era conocida por transmisión escrita, sino oral. Y, en la forma de transmitirla -en las guardas de un códice, incompleta y con mezclas dialectales-, tampoco parecían tener la intención de consignar por escrito un texto para darlo a conocer a un gran público lector.

Así pues, podemos considerar que el cantar antiguo presenta un marcado carácter de oralidad, pues contiene muchas de sus características. Ahora bien, si ello nos permite hablar de un texto oral "puro" o no, en sentido estricto, es una cuestión que puede quedar abierta. La razón para ello está en el hecho mismo de que nos haya llegado físicamente en un manuscrito. No obstante, un texto compuesto originariamente para transmisión oral, aun estando consignado en un manuscrito, puede mantener todos o casi todos sus rasgos de oralidad. En cuanto al cantar moderno, su claro carácter de transmisión escrita presenta quizá menos dudas, al carecer de los rasgos propios de la oralidad arriba comentados, además de la forma en que nos ha llegado consignado por escrito.

Finalmente, el estilo lingüístico de uno y otro texto de la historia de Hildebrando merecería un estudio aparte, el cual permitiría observar diferencias importantes entre el discurso en la literatura heroica de los siglos VIII-XI, por un lado, y el de una literatura de carácter cortesano-caballeresco, por otro. Por poner un ejemplo, pienso que sería muy productivo analizar el discurso de Hadubrando en uno y otro cantar. Ciertamente, esta tarea excede el objetivo y la temática del presente trabajo, pero considero que dicho análisis también podría arrojar unos resultados muy interesantes. 


\section{OBRAS CITADAS}

Adams, Kenneth. "The Metrical Irregularity of the Cantar de Mio Cid: A Restatement Based on the Evidence of Names, Epithets and Some Other Aspects of Formulaic Diction”. Bulletin of Hispanic Studies 49.2 (1972): 109-19.

Aguirre, José M. "Épica oral y épica castellana: tradición creadora y tradición repetitiva". Romanische Forschungen 80.1 (1968): 13-43.

Andersson, Theodore M. "Die Oral-Formulaic Poetry im Germanischen". Heldensage und Heldendichtung im Germanischen. Ed. Heinrich Beck. Berlin/New York: De Gruyter, 1988. 1-14.

Anónimo. Beowulfy otros poemas épicos antiguo germánicos. Ed. Luis Lerate. Barcelona: Seix Barral, 1974.

Anónimo. "Cantar de Hildebrand". Textos alemanes primitivos. La Edad Media temprana alemana en sus testimonios literarios. Ed. Berta Raposo. Valencia: Universitat de València, 1999. 158-69.

Anónimo. "Das Hildebrandslied". Althochdeutsches Lesebuch. Eds. Wilhelm Braune y Ernst A. Ebbinghaus. 17. a ed. Tübingen: Niemeyer, 1994. 84-85.

Anónimo. "Das Hildebrands Lied oder der Vater mit dem Sohn". Das Heldenbuch in der Ursprache. Vol. 2. Eds. Friedrich H. von der Hagen y Alois Primisser. Berlin: Reimer, 1825. 219-21.

Anónimo. "Das Hildebrandslied". Traducción inédita de Félix Díaz. La literatura alemana a través de sus textos. Ed. Luis A. Acosta. Madrid: Cátedra, 1997. 27-29.

Anónimo. Heliand und Genesis. Eds. Otto Behaghel y Burkhard Taeger. 10. ${ }^{a}$ ed. Tübingen: Niemeyer, 1996.

Anónimo. "Wessobrunner Gebet". Althochdeutsches Lesebuch. Eds. Wilhelm Braune y Ernst A. Ebbinghaus. 17. a ed. Tübingen: Niemeyer, 1994. 8586.

Anónimo. Zwei Fornaldarsögur (Hrólfssaga Gautrekssonar und Ásmundarsaga Kappabana) nach Cod. Holm. 7, 4to. Ed. Ferdinand Detter. Halle: Niemeyer, 1891.

Ashby-Beach, Genette. The Song of Roland: a Generative Study of the Formulaic Language in the Single Combat. Amsterdam: Rodopi, 1985.

Bäuml, Franz H. "The Oral Tradition and Middle High German Literature". Oral Tradition 1.2 (1986): 398-445.

Bergmann, Rolf, ed. Althochdeutsche und altsächsische Literatur. Berlin/Boston: De Gruyter, 2013. 
Boers, Frank, Seth Lindstromberg y June Eyckmans. "Is alliteration mnemonic without awareness-raising?”. Language Awareness 23.4 (2014): 291-303.

Chafe, Wallace L. "Integration and involvement in speaking, writing and oral literature". Spoken and Written Language: Exploring Orality and Literacy. Ed. Deborah Tannen. Norwood: Ablex Publishing, 1982. 35-53.

Classen, Albrecht. "The füngeres Hildebrandslied in its Early Modern printed versions: A contribution to fifteenth- and sixteenth-century reception history". Fournal of English and Germanic Philology 95.3 (1996): 359-81.

Curschmann, Michael. "Nibelungenlied und Nibelungenklage. Über Mündlichkeit und Schriftlichkeit im Prozess der Episierung". Deutsche Literatur im Mittelalter. Kontakte und Perspektiven. Hugo Kubn zum Gedenken. Ed. Christoph Cormeau. Stuttgart: Metzler, 1979. 85-119.

Curschmann, Michael. "The Concept of the Oral Formula as an Impediment to Our Understanding of Medieval Oral Poetry". Medievalia et Humanistica. Studies in Medieval \& Renaissance Cultture. Ed. Paul M. Clogan. Vol. 8. Cambridge: Cambridge UP, 1977. 63-76.

Curschmann, Michael. "Oral Poetry in Mediaeval English, French and German Literature: Some Notes on Recent Research”. Speculum 42 (1967): 382-89.

De Boor, Helmut. "Die nordische und deutsche Hildebrandsage". Zeitschrift für deutsche Philologie 49 (1923): 149-81.

Duggan, Joseph. "Formulaic Diction in the Cantar de Mio Cid and the Old French Epic”. Forum for Modern Language Studies 10.3 (1974): 260-69.

Dunphy, R. Graeme. "Orality". Camden House History of German Literature. Eds. Brian Murdoch y Malcolm Read. Vol. 1: Early Germanic Literature and Culture. Rochester: Camden House, 2004. 103-18.

Düwel, Klaus, y Nikolaus Ruge. "Hildebrandslied". Althochdeutsche und altsächsische Literatur. Ed. Rolf Bergmann. Berlin/Boston: De Gruyter, 2013. 171-83.

Düwel, Klaus. "Hildebrandsl Düwel Düwel ied". Die deutsche Literatur des Mittelalters: Verfasserlexikon. Eds. Kurt Ruh, Gundolf Keil, Werner Schröder, Burghart Wachinger y Franz Josef Worstbrock. Vol. 3. Berlin/ New York: De Gruyter, 1981. 1240-256.

Ebel, Uwe. "Historizität und Kodifizierung: Überlegegungen zu einem zentralen Aspekt des germanischen Heldenlieds". Althochdeutsch. Eds. Rolf Bergmann, Heinrich Tiefenbach y Lothar Voetz. Heidelberg: Carl Winter, 1987. 685-714. 
Foley, John M., y Peter Ramey. "Oral Theory and Medieval Literature”. Medieval Oral Literature. Ed. Karl Reichl. Berlin/Boston: De Gruyter, 2016. 71-102.

Foley, John M. "The textualization of South Slavic oral epic and its implications for oral-derived epic". Textualization of Oral Epics. Ed. Lauri Honko. Berlin/New York: Mouton de Gruyter, 2000. 71-87.

Foley, John M. Oral-Formulaic Theory and Research: An Introduction and Annotated Bibliography. New York: Garland, 1985.

Foley, John M. "Literary art and oral tradition in Old English and Serbian poetry". Anglo-Saxon England 12 (1983): 183-214.

Foley, John M. "Introduction: The Oral Poetry in Context". Oral Traditional Literature: A Festschrift for Albert Bates Lord. Ed. John M. Foley. Columbus: Slavica Publishers, 1981. 27-102.

Ford, John C. "Oral elements as literary skeuomorphs in the Middle English verse romances". Transitional Texts: Drifting between the Oral and the Written. Eds. S. Gintsburg, J. Ford \& A. Barandiaran. Número monográfico de Rilce 36.4 (2020): 1275-323.

Fry, Donald K. "Formulaic theory and Old English poetry". Report of the Twelfth Congress, Berkeley 1977. Eds. Daniel Heartz y Bonnie Wade. Kassel: Bärenreiter, 1981. 169-73.

Fry, Donald K. "Old English formulaic themes and type scences". Neophilologus 52 (1968): 48-54.

Fry, Donald K. “Old English formulas and systems”. English Studies 48 (1967): 193-204.

Green, Donald C. "Formulas and Syntax in Old English Poetry: A Computer Study". Computers and the Humanities 6.2 (1971): 85-93.

Greenfield, Stanley. "The Formulaic Expression of the theme of 'Exile' in Anglos-Saxon Poetry”. Speculum 30.2 (1955): 200-06.

Hackett, W. Mary. "Le style formulaire dans 'Girart de Roussillon”. Mélanges de langue et de littérature médiévales offerts à Pierre Le Gentil. Eds. Jean Dufournet y Daniel Poirion. Paris: SEDES \& CDU réunis, 1973. 345-52.

Haferland, Harald. "Das 'Nibelungenlied' im Zwischenbereich von Mündlichkeit und Schriftlichkeit". Zeitschrift für deutsches Altertum und deutsche Literatur 148.1 (2019): 28-84.

Harris, Joseph. "Old Norse Memorial Discourse between Orality and Literacy". Along the Oral-Written Continuum. Types of Texts, Relations and their Implications. Ed. Slavica Ranković. Turnhout: Brepols, 2010. 119-33. 
Harris, Joseph. "Eddic Poetry as Oral Poetry". Edda: A Collection of Essays. Eds. Robert J. Glendinning y Haraldur Bessason. Winnipeg: University of Manitoba, 1983. 210-42.

Haymes, Edward R. "Oral Theory and Medieval German Poetry". Oral Tradition 18.2 (2003): 258-60.

Heinzle, Joachim. Einführung in die mittelhochdeutsche Dietrichepik. Berlin/Nueva York: De Gruyter, 1999.

Heusler, Andreas. "Das alte und das junge Hildebrandslied". Preußische Fabrbücher 208 (1927): 143-52.

Hottenstein, Gertrud. "Ein Vergleich zwischen altem und jungem Hildebrandslied". Akten des 6. Internationalen Germanisten-Kongresses Basel 1980. Eds. Heinz Rupp y Hans-Georg Roloff. Vol. 2. Bern/Frankfurt am Main: Peter Lang. 237-42.

Jones, Alison. "Daniel and Azarias as evidence for the Oral-Formulaic carachter of Old English poetry". Medium Avum 35.2 (1966): 95-102.

Kartschoke, Dieter. Geschichte der deutschen Literatur im frühen Mittelalter. 3., aktualisierte Auflage. München: Deutscher Taschenbuch, 2000.

Kellogg, Robert. "Literacy and Orality in the Poetic Edda". Vox intexta. Orality and Textuality in the Middle Ages. Eds. Alger N. Doane y Carol B. Pasternack. Madison: The University of Wisconsin, 1991. 89-101.

Kienast, Walther. "Altes Hildebrandslied, Thidrekssaga und Junges Hildebrandslied". Herrings Archiv für das Studium der neueren Sprachen 76 (1922/23): 155-69.

Kiparski, Paul. "Oral poetry: Some linguistic and typological considerations". Oral Literature and the Formula. Eds. Benjamin A. Stolz y Richard S. Shannon. Ann Arbor: The Center for the Coordination of Ancient and Modern Studies, 1976. 73-106.

Lea, Brooke, David N. Rapp y Andrew Elfenbein. "Sweet Silent Thought. Alliteration and Resonance in Poetry Comprehension". Phychological Science 19.7 (2008): 709-16.

Lönnroth, Lars. "Iorð fannz œva né upphimin: A formula analysis". Speculum Norrenum: Norse Studies in Memory of Gabriel Turville-Petre. Ed. Ursula Dronke. Odense: University Press, 1981. 310-27.

Lönnroth, Lars. "Hjálmar's Death-Song and the Delivery of Eddic Poetry". Speculum 46.1 (1971): 1-20.

Lord, Albert B. The Singer Resumes the Tale. Ed. Mary L. Lord. Ithaca/London: Cornell University, 1995. 
Lord, Albert B. The Singer of Tales. Eds. Stephen Mitchell y Gregory Nagy. 2. ${ }^{a}$ ed. Cambridge/London: Harvard UP, 2000.

Magoun, Francis P. "The oral-formulaic character of Anglo-Saxon narrative poetry". Speculum 28.3 (1953): 446-67.

Meyer, Richard M. Die altgermanische Poesie nach ibren formelhaften Elementen. Berlin: Wilhelm Hertz, 1889.

Miklautsch, Lydia. "Fingierte Mündlichkeit? Zum Prolog des Wolfdietrich D". Neophilologus 86 (2002): 87-99.

Miletich, John S. "The Quest for 'the Formula': A Comparative Reappraisal”. Modern Pbilology 74.2 (1976): 111-23.

Minkova, Donka. Alliteration and Sound Change in Early English. Cambridge: University Press, 2003.

Mitchell, Stephen A. "The Sagaman and Oral Literature: The Icelandic Traditions of Hjörleifr inn kvensanni and Geirmundr heljarskinn". Comparative Research on Oral Traditions: A Memory for Milman Parry. Ed. John M. Foley. Columbus: Slavica Publishers, 1987. 395-423.

Müller, Jan-Dirk. "Medieval German Literature: Literacy, Orality and SemiOrality”. Medieval Oral Literature. Ed. Karl Reichl. Berlin/Boston: De Gruyter, 2012. 295-334.

Murray, Alan V. "The Livonian Rhymed Cbronicle as a Transitional Text: Formulaic Language in a Middle High German Verse Chronicle". Transitional Texts: Drifting between the Oral and the Written. Eds. S. Gintsburg, J. Ford \& A. Barandiaran. Número monográfico de Rilce 36.4 (2020): 1324-43.

Murray, Alan V. "Formulaic Language in the Livonian Rhymed Chronicle. Set Phrases and Discourse Markers in Middle High German History Writing”. Amsterdamer Beiträge zur Älteren Germanistik 79 (2019): 86-105.

Nagler, Michael N. "Towards a generative view of the oral formula". Transactions of the American Philological Association 98 (1967): 269-311.

O'Brien O'Keeffe, Katherine. "Orality and Literacy: The Case of AngloSaxon England". Medieval Oral Literature. Ed. Karl Reichl. Berlin/Boston: De Gruyter, 2012. 121-40.

Ochs, Elinor. "Planned and Unplanned Discourse". Syntax and Semantics. Discourse and Snyntax. Ed. Talmy Givón. New York/San Francisco/London: Academic Press, 1979. 51-80.

Parks, Ward. "The traditional narrator and the 'I heard' formulas in Old English poetry". Anglo-Saxon England 16 (1987): 45-66. 
Parry, Milman. L'épithète traditionnelle dans Homère: Essai sur un problème de style bomérique. Paris: Les Belles Lettres, 1928a.

Parry, Milman. Les formules et la métrique d'Homère. Paris: Les Belles Lettres, $1928 b$.

Parry, Milman. "Studies in the Epic Technique of Oral Verse-Making. I. Homer and Homeric Style". Harvard Studies in Classical Pbilology 41 (1930): 73-147.

Renoir, Alain. "The armor of the 'Hildebrandslied': An Oral-Formulaic point of view". Neuphilologische Mitteilungen 78.4 (1977): 389-95.

Renoir, Alain. A Key to Old Poems: The Oral-Formulaic Approach to the Interpretation of West-Germanic Verse. University Park/London: The Pennsylvania State University Press, 1988.

Riedinger, Anita. "The Old English Formula in Context". Speculum 60.2 (1966): 294-317.

Rosenfeld, Hellmut. "Das Hildebrandslied. Die indogermanischen VaterSohn-Kampf-Dichtungen und das Problem ihrer Verwandtschaft". Deutsche Vierteljabrsschrift für Literaturwissenschaft und Geistesgeschichte 26 (1952): 413-32.

Rumble, Thomas C. "The Hyran-Gefrignan Formula in Beowulf". Annuale Mediaevale 5 (1964): 13-20.

Russom, Geoffrey. Beowulf and Old Germanic Metre. Cambridge: Cambridge UP, 1998.

Rychner, Jean. La chanson de geste. Essai sur l'art épique des jongleurs. Genève: Droz, 1955.

Schaefer, Ursula. Vokalität: Altenglische Dichtung zwischen Mündlichkeit und Scbriftlichkeit. Tübingen: Narr, 1992.

Schneider, Karl. "Zum Hildebrandslied 37/38 und 49". Althochdeutsch. Eds. Rolf Bergmann, Heinrich Tiefenbach y Lothar Voetz. Heidelberg: Carl Winter, 1987. 655-69.

Schröder, Werner. "Hadubrands tragische Blindheit und der Schluß des Hildebrandsliedes". Deutsche Vierteljahrsschrift für Literaturwissenschaft und Geistesgeschichte 37.4 (1963): 481-97.

Schwarz, Werner. "Notes on formulaic expressions in Middle High German Poetry". Medieval German Studies Presented to Frederick Norman, Professor of German in the University of London, by his Students, Colleagues and Friends on the Occasion of his Retirement. Ed. Institute of Germanic Linguistics. London: University of London, 1965. 60-70. 
Tannen, Deborah. "Oral and Literate Strategies in Spoken and Written Narratives”. Language 58.1 (1982): 1-21.

Waltman, Franklin M. "Formulaic Expression and Unity of Autorship in the Poema of Mio Cid". Hispania 56.3 (1973): 569-78.

Whallon, William. "Formulas for Heroes in the 'Illiad' and in 'Beowulf'". Modern Philology 63.2 (1965): 95-104.

Webber, Ruth H. "Formulaic language in the 'Mocedades de Rodrigo"'. Hispanic Review 48.2 (1980): 195-211.

Webber, Ruth H. "Formulistic Diction in the Spanish Ballad". University of California Publications in Modern Philology 34 (1951): 175-277.

Windelberg, Marjorie, y Gary Miller. "How (Not) to Define the Epic Formula". Olifant 8.1 (1980): 29-50.

Wishard, Armin. "Formulaic Composition in the Spielmannsepik". Papers on Language \& Literature 8.3 (1972): 243-51.

Wolff, Ludwig. "Das jüngere Hildebrandslied und seine Vorstufe". Hessische Blätter für Volkskunde 39 (1941): 54-63. 


\section{Section II: The Contemporary Maghreb and South Arabia}


